\title{
Acoustic recognition of aircraft types in flight
}

\author{
Ichiro Yamada, Akinori Yokota, Kohei Yamamoto, and Susumu Shimizu \\ Kobayasi Institute of Physical Research, \\ 3-20-41, Higashimotomachi, Kokubunji, Tokyo, 185 Japan
}

(Received 4 June 1984)

\begin{abstract}
A method for the acoustic recognition of aircraft types and flight patterns is presented, in which a discriminatory analysis slightly different from the ordinary one is used and peak-hold spectra (one-third octave band) relative to the peak value of A weighted sound pressure levels are selected for characteristic parameters of reference patterns. The newly developed procedure proved to be able to recognize aircraft types and flight patterns accurately irrespective of the mission of an airfield. Only a small number of parameters are required in the procedure.
\end{abstract}

PACS number: 43. 50. Yw, 43. 60. Gk

\section{INTRODUCTION}

Noise exposure resulting from aircraft operations occupies a very important part in all noise pollution problems which have strong influences on the living environment. Field measurements have been frequently carried out in order to evaluate the aircraft noise exposure. But one can usually continue measurements for only a week or more at most, and it is rather difficult to estimate long-term effects of aircraft noise on the environment. Therefore, noise monitoring systems are often set up around airports. In such systems it is desirable to manage automatically not only with the discrimination of aircraft noise from other environmental noises, but also with the recognition of the aircraft type and flight pattern. The former has already been realized successfully by an acoustic method using space-time correlation functions of aircraft noise ${ }^{1,2)}$ in addition to threshold values of a peak level and a duration time of fly-over noise. On the other hand, the latter has not been necessarily well developed up to the present. The DTAX information i.e., Domestic Telecommunication Automatic Exchange for aircraft operations may be utilized. However, this information is not always available and sometimes does not correspond well to observations of aircraft noise around the airport.

So, it is desired to develop an acoustic procedure for identifying the aircraft type and flight pattern using aircraft noise itself, which was observed at each local noise monitoring station. In several works of Mizutani et al., ${ }^{3-5)}$ they used not only narrow constant-bandwidth band spectra, but also constant percentage bandwidth band spectra (octave or one-third octave band spectra) of aircraft noise, when the distance between the aircraft and observation point became minimum, or when the maximum noise level was observed. They adopted a rather complex classification rule to produce some acceptable results in their recognition procedure. ${ }^{5}$ )

In this paper a new recognition method is introduced. It is based on a simple and efficient principle and is able to be realized as a real-time processor in a cheap and compact hardware. Measurements of fly-over noise for various types of aircraft have been made beneath the flight path near the airport. Discriminatory analysis based on one-third octave band spectra showed a fairly good possibility of the recognition of aircraft types in flight acoustically.

\section{RECOGNITION PROCEDURE}

The recognition problem of the aircraft type can be viewed as an exercise of statistical discriminatory 
analysis. First of all, it is necessary to make noise measurements for various types and flight patterns of aircraft that will be expected to be observed at a station. Noise data are analysed to extract appropriate characteristic parameters. They are clustered to several groups, which is expressed as "type-groups" in the following discussion, according to aircraft types, flight patterns, characteristics of noise exposure et cetera. Then they are averaged to create reference patterns for respective type-groups. This process will be discussed in chapter 3 .

Let us describe a procedure assigning an observed aircraft noise signal to one of type-groups according to the flow chart shown in Fig. 1. Here, it is assumed that there are $M$ type-groups and their reference pattern vectors are $\overrightarrow{\boldsymbol{P}}_{i}=\left(p_{1 i}, p_{2 i}, \cdots, P_{N i}\right), i=$ $1,2, \cdots, M$ where $p_{n i}$ expresses the $n$-th parameter for $i$-th group. When a fly-over noise sample of unknown aircraft type is observed, its sample pattern vector of noise characteristics, $\vec{X}=\left(x_{1}, x_{2}, \cdots, x_{N}\right)$ is estimated. Here $x_{n}$ represents $n$-th characteristic parameter for the sample. $\vec{X}$ is compared to reference patterns of respective type-groups $\vec{P}_{i}, i=1,2, \cdots, M$. To evaluate the similarity between the sample and each of reference patterns, a series of candidate distance scores $D_{1}\left(\vec{X}, \vec{P}_{i}\right), i=1,2, \cdots, M$ are computed according to the following definition of a weighted distance;

$$
\begin{aligned}
D_{1}\left(\vec{X}, \vec{P}_{i}\right)= & {\left[\sum_{n=1}^{N}\left(x_{n}-p_{n i}\right)^{2} \cdot W_{n i}\right]^{1 / 2}, } \\
& i=1,2, \cdots, M,
\end{aligned}
$$

where $W_{n i}$ is a weight of $n$-th parameter for $i$-th aircraft type. In this study, the weight $W_{n i}$ is simply defined as a reciprocal of the estimated variance of $n$-th parameter for $i$-th aircraft type. The sample is assigned to a type-group, the distance score of which is the smallest and below a pre-determined threshold value $D_{0}$. If the smallest score is greater than $D_{0}$, the sample is ascribed to an unknown aircraft other

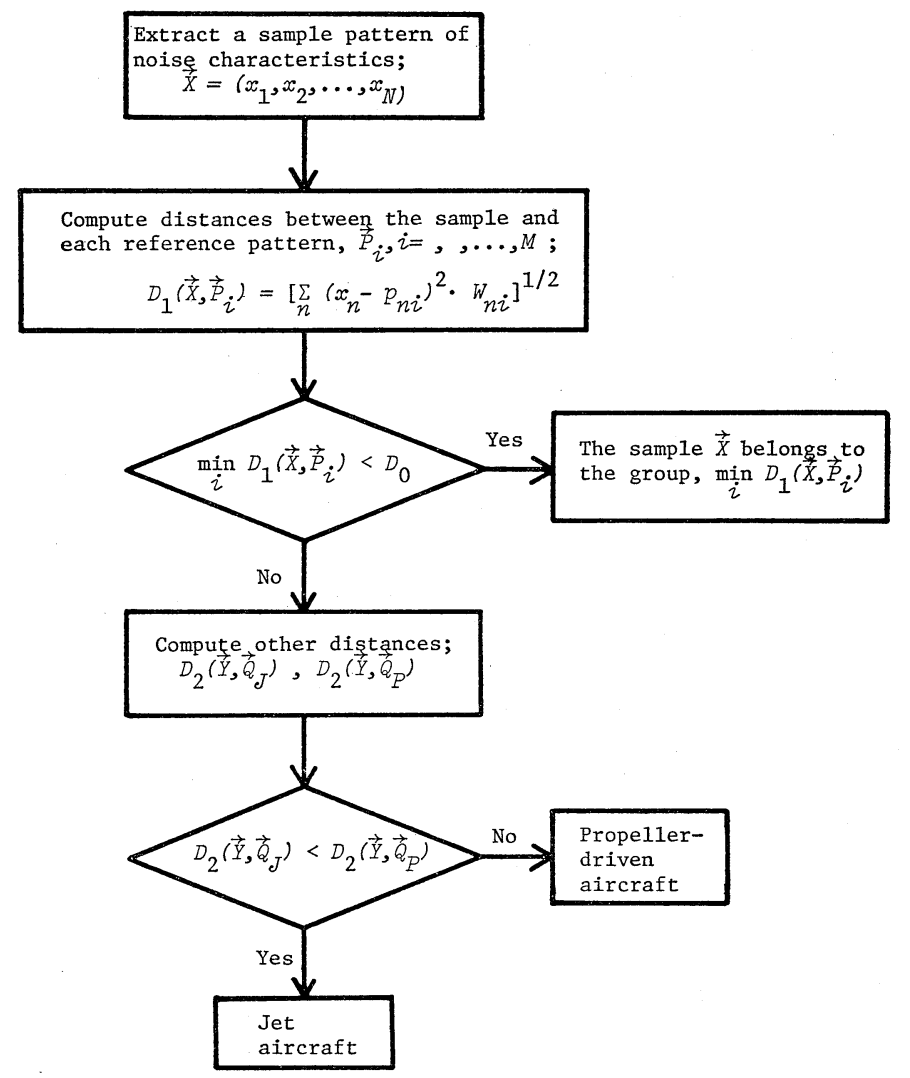

Fig. 1 A flow diagram classifying an observed aircraft noise. 


\section{YAMADA et al.: ACOUSTIC RECOGNITION OF AIRCRAFT TYPES IN FLIGHT}

than the pre-determined type-groups. Then proceeding to the second stage of the procedure, it is examined whether it belongs to jet aircraft or to propeller-driven aircraft. This stage is inevitable because various unexpected types of aircraft often fly over at the airfield. Distance scores $D_{2}\left(\vec{Y}, \vec{Q}_{j}\right)$, $j=J, P$ are calculated from a different sample pattern $\vec{Y}$ and different reference patterns $\vec{Q}_{j}, j=J, P$. The definition of $D_{2}\left(\vec{Y}, \vec{Q}_{j}\right), \vec{Y}$ and $\vec{Q}_{j}$ are respectively the same as $D_{1}\left(\vec{X}, \vec{P}_{i}\right), \vec{X}$ and $\vec{P}_{i}$.

The recognition procedure defined above is slightly different from the ordinary discriminatory analysis. $\left.{ }^{6}, 7\right)$ If we obey the ordinary analysis, type-groups are usually assumed to be correspondent to different populations respectively and every sample should necessarily be classified into any of type-groups that are prescribed in advance. If it is assumed that all populations have multivariate normal distributions and that their covariance matrices are the same, any sample is discriminated to belong to a group that has the maximum likelihood function. Taking a logarithm of the likelihood, since logarithm is a monotonic function, the ordinary classification rule can be written as follows;

a sample $\vec{X}$ is assigned to a group $\vec{P}_{i}$ such as

$$
D D\left(\vec{X}, \vec{P}_{i}\right)<D D\left(\vec{X}, \vec{P}_{j}\right) \quad \text { for all } j \neq i
$$

where

$$
D D\left(\vec{X}, \vec{P}_{i}\right)=\left[\left(\vec{X}-\vec{P}_{i}\right)^{t} \cdot(W)^{-1} \cdot\left(\vec{X}-\vec{P}_{i}\right)\right]^{1 / 2},
$$

$W$ is the covariance matrix, $(W)^{-1}$ is the inverse of the matrix $W$, and the superscript $\mathrm{t}$ indicates the transpose of a vector. The quantity $\operatorname{DD}\left(\vec{X}, \vec{P}_{i}\right)$ can be called the general distance between the unknown sample $\vec{X}$ and the reference $\vec{P}_{i}$. That is, the sample is simply classified on the basis of a minimum distance rule. If all parameters show no significant inter-parameter correlations each other and have the same variance, $D D\left(\vec{X}, \vec{P}_{i}\right)$ comes to the commonly used Euclidean distance.

Equation (1) includes no covariance term and the variances utilized vary as a function of type-groups. That is, in our formulation, it is assumed that all populations of type-groups have their own covariance matrices but there is no inter-correlation between characteristic parameters. Contrary to it, in the ordinary formulation of Eq. (3), all populations are assumed to have the same covariance matrix, which is computed as the average over all populations. In this respect, Eq. (1) differs from Eq. (3). The validity of using Eq. (1) will be discussed in section 3.4 from an experimental result. From a practical point of view, it is certain that the formulation of Eq. (1) is simple and appropriate for the hardware realization of an automatic acoustic recognition system of the aircraft type and flight pattern. From the definition of the distance of Eq. (1), when another noise characteristic parameter is desired to be added to the constituents of pattern vectors, it is needed only to estimate the average and variance of the new parameters for each type-group. Furthermore, if the variance on some parameter of certain aircraft type-group is very large, the weight value becomes too small to affect the distance. So, if some type-group is clearly determined by only a few parameters, weights of all other parameters can be suppressed, from the beginning, to be so small values as not to affect the computation of the distance.

\section{PROCEDURES TO SELECT CHARACTERISTIC PARAMETERS}

\subsection{Collection of Aircraft Noise Data}

Field measurements of aircraft noise were made in order to investigate what kinds of characteristics were applicable to aircraft type recognition and how to constitute reference patterns. Aircraft types and flight patterns vary with the mission of an airfield. First, Yokota military airbase was chosen. This airfield is located on the outskirts of Tokyo. Measurements were made consecutively for five days at two sites close to the runway, that is, about $700 \sim 800 \mathrm{~m}$ from both ends of the runway (see the geometry of microphone locations in Fig. 2). At each site four sound level meters were set, two of which were raised at a height of $1.2 \mathrm{~m}$ above the ground and others were laid just on the ground. Two infra-sonic microphones were also set on the ground. Using such measuring devices and a 8-channel FM tape recorder, noise data were recorded in the audiofrequency range through $\mathrm{C}$ weighting circuit and infra-sonic sound in the frequency range of $0.4 \mathrm{~Hz} \sim$ $1 \mathrm{kHz}$.

Although various types of aircraft flying at the airfield can be observed, it is decided to classify aircraft types and fiight patterns to only nine groups shown in Table 1 i.e., Take-off (T/O) and Landing (L/D) of three main aircraft types, helicopter and others 

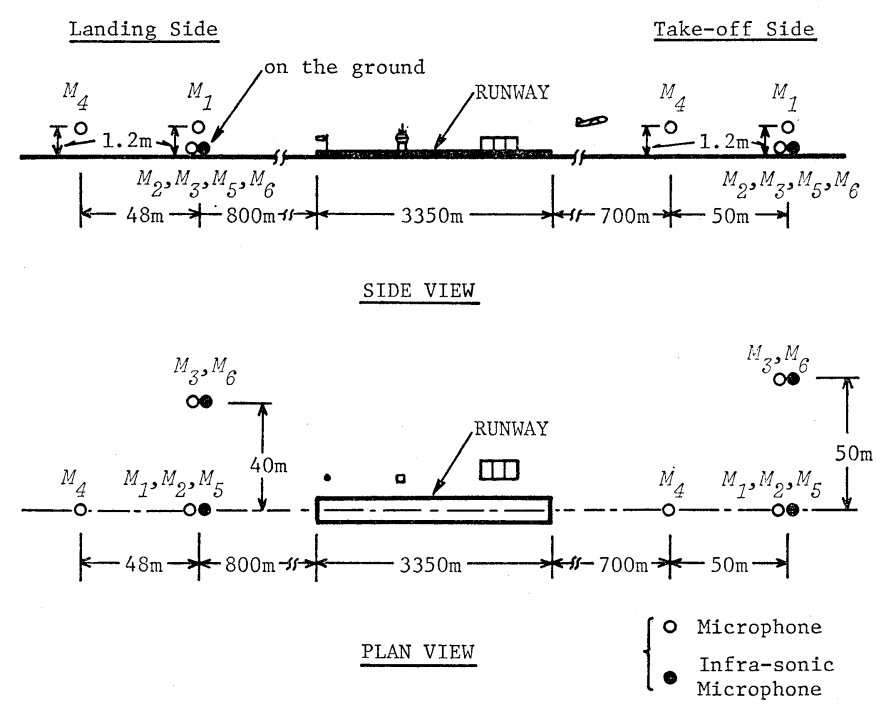

Fig. 2 Geometrical locations of microphones in field measurements in the vicinity of Yokota airfield, Tokyo.

Table 1 A classification of type-groups used in the exercise of the recognition procedure for Yokota data.

1 Take-off noise of C-141

(4-engine large turbo-fan airplane)

$2 \quad$ Landing noise of C-141

(4-engine large turbo-fan airplane)

3 Take-off noise of T-39

(twin-engine small turbo-fan training plane)

4 Landing noise of T-39

(twin-engine small turbo-fan training plane)

5 Take-off noise of C-130

(4-engine large turbo-prop airplane)

6 Landing noise of C-130

(4-engine large turbo-prop airplane)

7 Helicopter

8 Other jet airplane

9 Other propeller-driven airplane

(jet airplane and propeller-driven airplane) from taking into consideration about frequency distributions of observations of each aircraft type through a year. C-130 and T-39 frequently repeat Touch \& Go $(\mathrm{T} / \mathrm{G})$ operations in addition to the normal $\mathrm{T} / \mathrm{O}$ and $L / D$. These $T / G$ operations were treated to be included in the normal $\mathrm{T} / \mathrm{O}$ or $\mathrm{L} / \mathrm{D}$ operations in the recognition procedure. They are, however, considered to be executed as practices of flight proce- dures for pilots, and so their flight patterns and noise exposures vary to a great extent. For example, the height of aircraft varied from several tens to hundreds meters above the ground at the Take-off site during the field measurements. Note that the noise generated by a helicopter possesses a strong directivity in its characteristic low frequency component i.e., Blade-Slap noise, and it was very difficult to observe the whole time history of level fluctuations.

\subsection{Data Analysis}

It seems to be certain that various information expressing differences among aircraft types and flight patterns is included in time histories of power spectra of aircraft noise. So, noise data were analysed through a real time one-third octave band spectrum analyser, where the time constant of averaging was SLOW. Then they were sampled by a time rate of $0.1 \mathrm{~s}$, and were taken into a mini-computer, at least, over a time span that the sound pressure level was maintained above a level $10 \mathrm{~dB}$ lower than the maximum A weighted sound pressure level, which is called as "dBA-peak" in the following discussion. In data analyses, 30 one-third octave band-pass filters were used besides $\mathrm{A}$ and $\mathrm{C}$ weighting networks. Midband frequencies of those band-pass filters were $25 \mathrm{~Hz} \sim 20 \mathrm{kHz}$. Five bands above $8 \mathrm{kHz}$ were not used because of low signalto-noise ratio. 


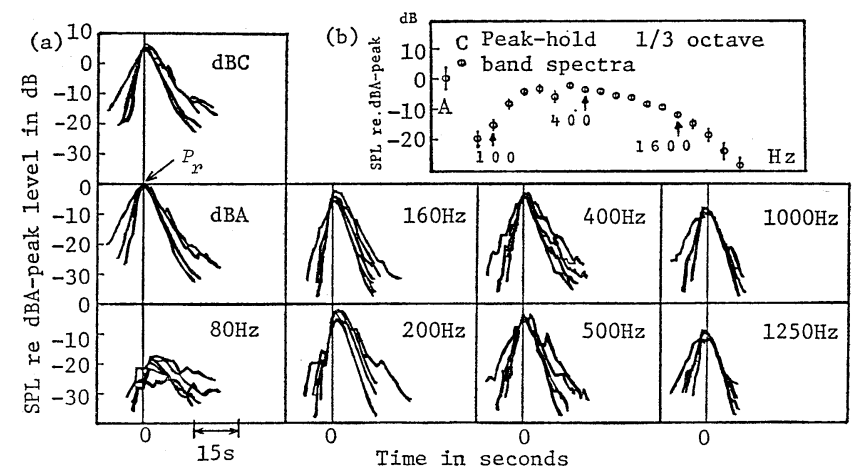

Fig. 3 (a) An example of superpositions of time histories of one-third octave band spectra of aircraft fly-over noise so that peak positions of A weighted sound pressure levels should coincide with a position $P_{\mathrm{r}}$ designated previously. (b) Average peak-hold spectra (one-third octave band) and their confidence intervals. Lines expressing confidence intervals $( \pm \sigma$; standard deviation) are drawn vertically around average values. $\pm \sigma$ were computed after normalized by dBA-peak levels. But the confidence interval for A weighted sound level i.e., dBA-peak shows the standard deviation of dBA-peak level itself.

The variation in sound pressure levels of aircraft noise was very large. So, time histories of one-third octave band spectra were piled up for each aircraft type so that dBA-peak positions should coincide with a position $\boldsymbol{P}_{\mathrm{r}}$ which was designated previously both in the level and time scale (see an example in Fig. 3). It turned out that peak positions in each onethird octave bands were quite stable both in the level and time scale among samples. From these relative band spectra normalized by dBA-peak, it is possible to extract a lot of promising characteristics available for recognition parameters. Averages and variances of following characteristics were computed; (1) peak values of band levels relative to the level of dBA-peak, (2) time differences between dBA-peak and peak positions of band levels, and (3) duration times overstepping a level $10 \mathrm{~dB}$ below the peak level in every one-third octave band.

Figure 4 shows differences of spectral shapes at three instants of a time history observed during a T-39 T/O flying-over. One can see that a dip frequency moves as the aircraft flies over. Although characteristics (1) (3) stably express differences among various aircraft types and flight patterns, (2) and (3) may change with the flying speed or the distance from the aircraft to the observer. In other words, characteristics (2) and (3) are sensitive to the flight procedure and observation site location.

After all, it was decided to utilize relative peak
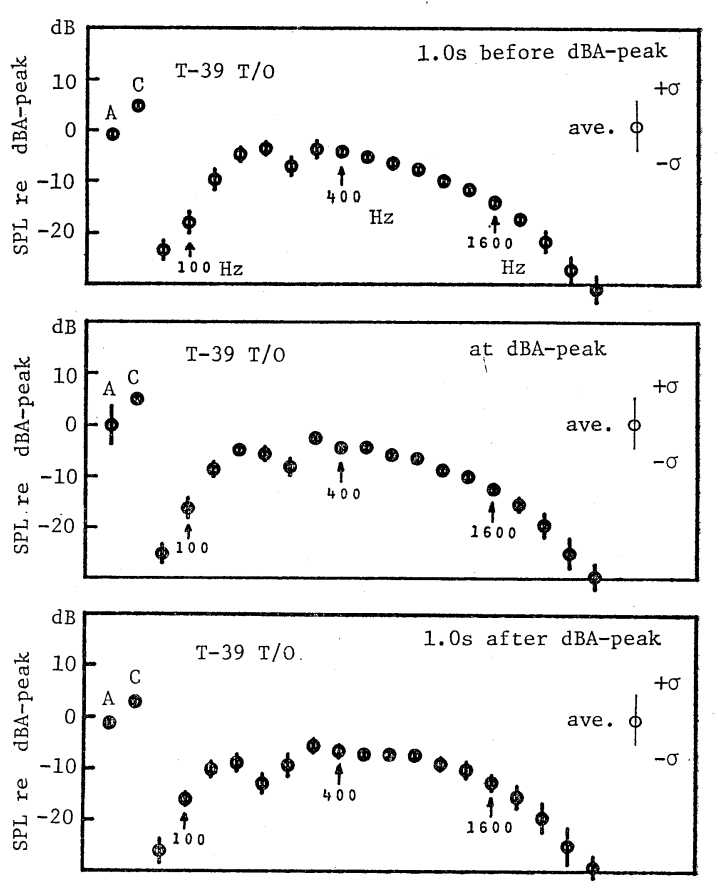

$1 / 3$ octave frequency band

Fig. 4 Changes of instantaneous one-third octave band spectra at three instants $(1.0 \mathrm{~s}$ before dBA-peak, at dBA-peak and $1.0 \mathrm{~s}$ after dBA-peak) of a time history observed during a T-39 fly-over at $M_{1}$. 
levels of various one-third octave bands during the aircraft fly-over as characteristics from which parameters to discriminate the aircraft type could be extracted. The peak level of a time history in each one-third octave band can be easily obtained only with simple and small hardwares such as bandpass filters, squaring device and peak-holding circuit, etc.

It was investigated if raising a microphone from the ground or changing its location would severely affect the acoustic recognition procedure. If a microphone is raised from the ground surface, reflections of sound waves cause multipath interferences and ripples are created in the observed noise spectra. ${ }^{8)}$ Frequencies of peaks and dips of such spectral rip-

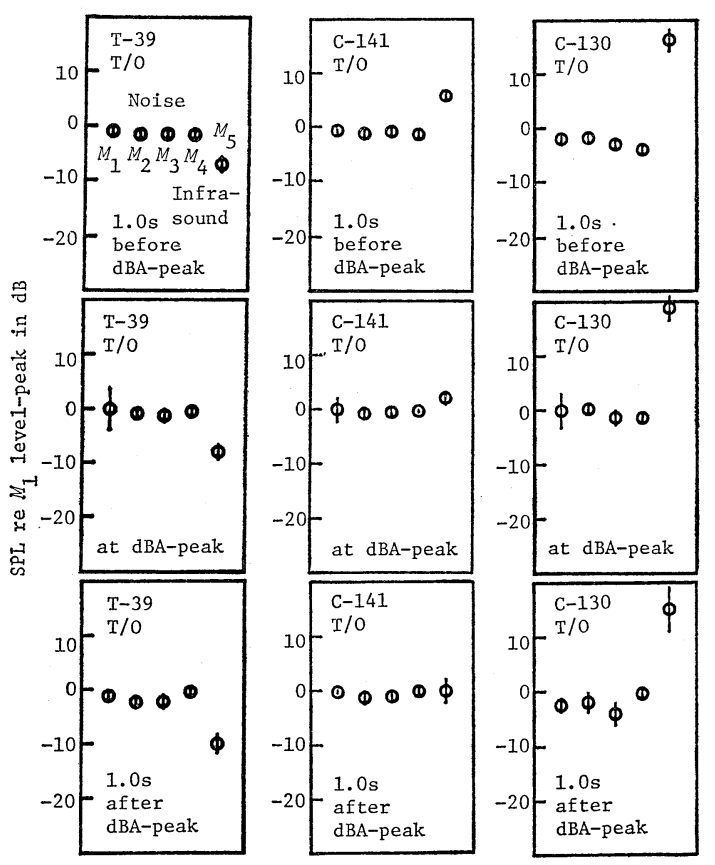

Fig. 5 Comparisons of instantaneous A weighted SPL $\left(M_{1} \sim M_{4}\right)$ and infrasonic SPL passed through a band pass filter (pass-band; $2.24 \sim 90 \mathrm{~Hz})\left(M_{5}\right)$ at three instants $(1.0 \mathrm{~s}$ before dBA-peak, at dBApeak and $1.0 \mathrm{~s}$ after dBA-peak) of time histories observed during aircraft fly-overs of T-39, C-141 and C-130. Vertical lines for $M_{2} \sim M_{5}$ show confidence intervals $\pm \sigma$ around average values after normalized by dBA-peak levels at $M_{1}$, but the lines for $M_{1}$ indicate standard deviations of $\mathrm{A}$ weighted SPL at $M_{1}$, themselves. ples will change with the incidence angle of sound ray when the aircraft flies over. Consequently, observed one-third octave band spectra may change their values from time to time, especially in low frequency bands. ${ }^{9}$ However, the output signal of each one-third octave band will be passed through a peak-holding circuit before extracting characteristic parameters of the recognition procedure, as is already stated above. Therefore, level fluctuations in one-third octave bands are expected to be alleviated because peaks and dips of the spectral ripple move over several bands during the aircraft fly-over. To confirm this expectation, time histories of A weighted sound pressure levels and infra-sonic sound pressure levels passed through a band-pass filter (pass-band was $2.24 \sim 90 \mathrm{~Hz}$ or $2.24 \sim 56 \mathrm{~Hz}$ ) were examined. As a result, it was found that values of observed dBApeaks relative to that of $M_{1}$ microphone were quite stable (Fig. 5) and differences were very small among microphones regardless of heights and locations of microphones. Results of a recognition procedure by raised microphones will be referred in a description for another experiment in chapter 4. On the other hand, peak values of infra-sonic sound pressure levels relative to dBA-peak changed with aircraft types, flight patterns and microphone locations. Moreover, infra-sonic sound pressure levels are often contaminated with wind generated noise. Therefore, such a low frequency component was determined to be used only for identifying the helicopter. It generates very strong impulsive noise components in low frequency bands i.e., Blade-Slap noise.

\subsection{Selection of Characteristic Parameters}

Considering the easiness of hardware realization and the insensitiveness of parameters to the microphone location on the basis of the analysis in section 3.2, characteristics from which recognition parameters were selected were limited to peak-hold spectra (onethird octave band) normalized relative to dBA-peak. Their averages and standard deviations were plotted for all aircraft types which were classified in Table 1 . Figure 6 (a) shows T/O patterns and Fig. 6 (b) for L/D. Although all frequency bands can be used to constitute a reference pattern, it is not efficient for the construction of a processor in a simple and cheap hardware. Parameters expressing differences among type-groups were selected so that it would be able to classify noise data as precise as possible with a small 


\section{YAMADA et al.: ACOUSTIC RECOGNITION OF AIRCRAFT TYPES IN FLIGHT}
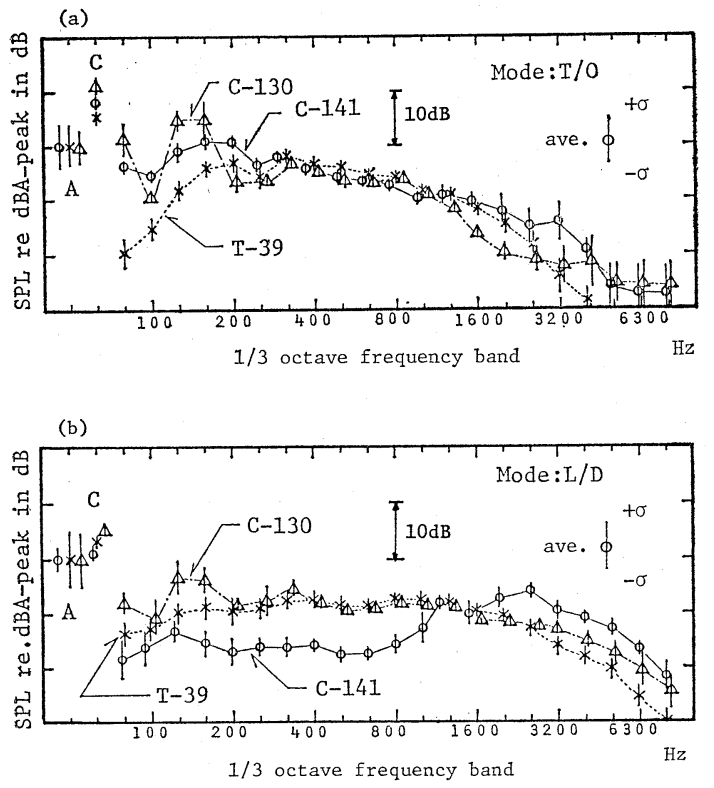

Fig. 6 Comparisons of average peak-hold spectra (one-third octave band) and their confidence intervals for aircraft types in Table 1; (a) T/O, and (b) L/D.

number of parameters. It is, however, not easy to select a set of effective parameters by a reasonable criterion of effectiveness, ${ }^{10)}$ for the recognition procedure adopted in this paper is composed of two stages to deal with various types of unknown aircraft other than pre-determined type-groupes. So, parameters were selected simply by visual inspection of average peak-hold characteristics. Only nine characteristic parameters were chosen (Table 2). Parameters $1 \sim 8$ are used in the first stage of the recognition procedure. Parameter 1 is selected especially for the discrimination of helicopter BladeSlap noise. On the other hand, the ninth parameter is used in the second stage to discriminate whether the examined aircraft is a propeller-driven aircraft or not. In general, noise spectra of jet aircraft spread over a wide frequency range and have smooth spectral shapes, while propeller-driven aircraft has a lot of peaks and dips in the spectra. To detect this difference, the ninth parameter i.e., a sum of square magnitudes of first order differences of spectra adjacent to each other over several frequency bands is computed by an equation,
Table 2 Characteristic parameters selected in the exercise for Yokota data.

$125 \mathrm{~Hz} 1 / 3$ octave band peak level re. dBA-peak

2 Difference of 50 and $63 \mathrm{~Hz} \mathrm{1/3}$ octave band peak levels

$380 \mathrm{~Hz} 1 / 3$ octave band peak level re. dBA-peak

$4125 \mathrm{~Hz} 1 / 3$ octave band peak level re. dBA-peak

$5 \quad 200 \mathrm{~Hz} 1 / 3$ octave band peak level re. dBA-peak

$6 \quad 315 \mathrm{~Hz} 1 / 3$ octave band peak level re. dBA-peak

$72500 \mathrm{~Hz} 1 / 3$ octave band peak level re. dBA-peak

8 C weighted SPL peak level re. dBA-peak

9 Squared sum of first order differences between $100 \sim 200 \mathrm{~Hz} 1 / 3$ octave band peak levels

$$
Q=\sum_{k=k_{1}}^{k_{2}}\left|L_{k+1}-L_{k}\right|^{2},
$$

where $L_{k}$ is $k$-th one-third octave band peak-hold sound pressure level. This single parameter $Q$ forms the discrimination parameter vector in this study.

As already stated in chapter 2, a pre-determined threshold value $D_{0}$ is used to judge if a sample belongs to any of pre-determined type-groups. The value was adjusted manually so that more reasonable classifications should be made between pre-determined type-groups and others.

The evaluation of averages and standard deviations of reference parameters was executed from a small number (approximately 5 10 samples) of typical observations that were obtained at normal $\mathrm{T} / \mathrm{O}$ and $\mathrm{L} / \mathrm{D}$ operations for each type-group. Extraordinary flight operations such as $\mathrm{T} / \mathrm{G}$, low-pass and high-pass flights were excluded from the computation. The result is shown in Table 3.

\subsection{Validity of Assumptions on the Distance Com- putation Rule}

In chapter 2, somewhat different distance computation rule of Eq. (1) was adopted for assigning an observed aircraft noise to one of pre-determined type-groups. The covariance matrix among parameters was estimated for each type-group in order to investigate if the assumptions supporting Eq. (1) are satisfied. The entire observations $(16 \sim 55$ observations for each type-group) of normal operations were used in this computation.

The diagonal components of the covariance matrices are equivalent to the weights $W_{i}$ in Eq. (1). One can see clearly from Table 3 that the parameters 
Table 3 Averages and their standard deviations of characteristic parameters in Table 2.

\begin{tabular}{crrrrrrrr}
\hline & \multicolumn{7}{c}{ Parameter } \\
\cline { 2 - 8 } Type & $25 \mathrm{~Hz}$ & $63 \mathrm{~Hz}-50 \mathrm{~Hz}$ & $80 \mathrm{~Hz}$ & $125 \mathrm{~Hz}$ & $200 \mathrm{~Hz}$ & $315 \mathrm{~Hz}$ & $2500 \mathrm{~Hz}$ & $\mathrm{dBC}-\mathrm{dBA}$ \\
\hline T-39 & -32.2 & 1.9 & -19.9 & -8.3 & -3.4 & -2.2 & -18.7 & 5.2 \\
T/O & 3.2 & 1.1 & 2.4 & 1.5 & 1.3 & 1.1 & 2.1 & 0.7 \\
\hline T-39 & -28.9 & 3.1 & -12.7 & -9.6 & -10.1 & -8.6 & -13.7 & 2.9 \\
L/D & 3.0 & 1.8 & 2.6 & 2.8 & 2.9 & 2.0 & 1.6 & 0.9 \\
\hline C-141 & -12.5 & -0.5 & -3.6 & -1.1 & 0.6 & -2.3 & -14.9 & 8.1 \\
T/O & 0.7 & 0.8 & 0.9 & 1.5 & 0.6 & 0.7 & 2.8 & 0.7 \\
\hline C-141 & -20.8 & -2.2 & -18.2 & -13.0 & -16.9 & -16.0 & -5.8 & 1.0 \\
L/D & 2.4 & 0.9 & 3.4 & 1.7 & 2.4 & 1.6 & 1.1 & 0.4 \\
\hline C-130 & -21.5 & 8.3 & 1.4 & 4.7 & -6.4 & -3.0 & -20.9 & 11.2 \\
T/O & 2.4 & 2.2 & 2.9 & 1.7 & 2.0 & 1.1 & 2.1 & 1.5 \\
\hline C-130 & -16.7 & 8.5 & -7.8 & -3.1 & -8.1 & -5.1 & -12.0 & 5.5 \\
L/D & 1.9 & 2.7 & 1.7 & 2.7 & 1.0 & 1.4 & 0.6 & 0.9 \\
\hline Heli. & 3.4 & 2.5 & 8.9 & 7.0 & -3.9 & -4.8 & -17.8 & 16.9 \\
& 1.2 & 0.8 & 4.7 & 4.6 & 3.6 & 5.7 & 4.7 & 4.7 \\
\hline
\end{tabular}

In each column, upper case shows the average and lower case, the standard deviation.

have rather different variances among type-groups. Therefore, it is difficult to assume that covariance matrices of all type-group populations are identical. Non-diagonal components of the covariance matrix mean inter-parameter correlations. To evaluate this relation clearly, covariance matrices were normalized to correlation coefficients. Several parameters showed significant inter-parameter correlations, where the correlation coefficient was greater than 0.7 .

As the assumptions on neither Eq. (1) nor Eq. (3) are satisfied, strictly speaking, it is desirable to provide an accurate covariance matrix for each typegroup. However, in most practical situations, it is difficult to use such a complicated distance computation rule, because the kinds of aircraft type-groups and their flight procedures vary according to the mission of an airfield and the site location of an observation point. As a result, a more permissible lightening of the restrictions is to omit inter-parameter correlation terms in the covariance matrix, that is, to adopt the distance definition of Eq. (1).

\section{RESULTS OF EXPERIMENTAL STUDIES}

First, the recognition procedure described in chapter 2 was applied to all noise data obtained in the field measurements stated above. Only nine characteristic parameters in Tables 2 and 3 were used. The threshold value $D_{0}$ for determining if a sample is ascribed to an unknown aircraft other than the previously designated type-groups was varied so that a good recognition result could be obtained. The result is shown as a confusion matrix in Table 4. Column classifications are true aircraft types observed and rows are recognized type-groups. One can see that a fairly good discrimination has been attained in spite of the small number of parameters. In wrong identifications, it is most conspicuous that $\mathrm{T} / \mathrm{O}$ and $\mathrm{L} / \mathrm{D}$ noise data of T-39 are incorrectly classified to other jet aircraft. These noise data are, however, mainly due to low-pass and high-pass flight operations and they should be treated as belonging to different type-groups.

The discrimination procedure based on the common Euclidean distance rule was applied to the same noise data. The rate of correct discriminations by Euclidean distance was approximately $10 \%$ lower than the result of Table 4. The rule defined by Eq. (3) was also applied to the data. The covariance matrix $W$ was determined as an average matrix over all of type-groups from the results in section 3.4. The magnitude of diagonal component of the inverse matrix $W^{-1}$ was a power of 10 greater than nondiagonal components. The result was, however, $5 \%$ worse than the Euclidean case. 


\section{YAMADA et al.: ACOUSTIC RECOGNITION OF AIRCRAFT TYPES IN FLIGHT}

Table 4 The result of recognition in a confusion matrix for Yokota data.

\begin{tabular}{|c|c|c|c|c|c|c|c|c|c|c|}
\hline \multirow{3}{*}{ In } & & \multicolumn{9}{|c|}{ Out } \\
\hline & & \multicolumn{2}{|c|}{ C-141 } & \multicolumn{2}{|c|}{ C-130 } & \multicolumn{2}{|c|}{ T-39 } & \multirow{2}{*}{ Heli. } & \multicolumn{2}{|c|}{ Others } \\
\hline & & $\mathrm{T} / \mathrm{O}$ & L/D & $\mathrm{T} / \mathrm{O}$ & $L / D$ & $\mathrm{~T} / \mathrm{O}$ & $L / D$ & & Jet & Prop. \\
\hline \multirow{2}{*}{ C-141 } & $\mathrm{T} / \mathrm{O}$ & 10 & & & & & & & 2 & \\
\hline & L/D & & 15 & & & & & & 1 & \\
\hline \multirow[t]{2}{*}{ C-130 } & $\mathrm{T} / \mathrm{O}$ & & & 45 & & & & & & \\
\hline & L/D & & & 1 & 25 & & 1 & & & 1 \\
\hline \multirow[t]{2}{*}{ T-39 } & $\mathrm{T} / \mathrm{O}$ & & & & & 55 & 1 & & 6 & 1 \\
\hline & L/D & & & & 2 & 2 & 41 & & 3 & \\
\hline Helicopter & & & & & & & & 33 & 3 & 1 \\
\hline \multirow[t]{2}{*}{ Others } & Jet & & 2 & & 1 & 5 & 4 & & 21 & 1 \\
\hline & Propeller & & & 3 & & & & & 1 & 17 \\
\hline
\end{tabular}

Column classifications are true aircraft types and flight patterns, and rows show recognized type-groups.
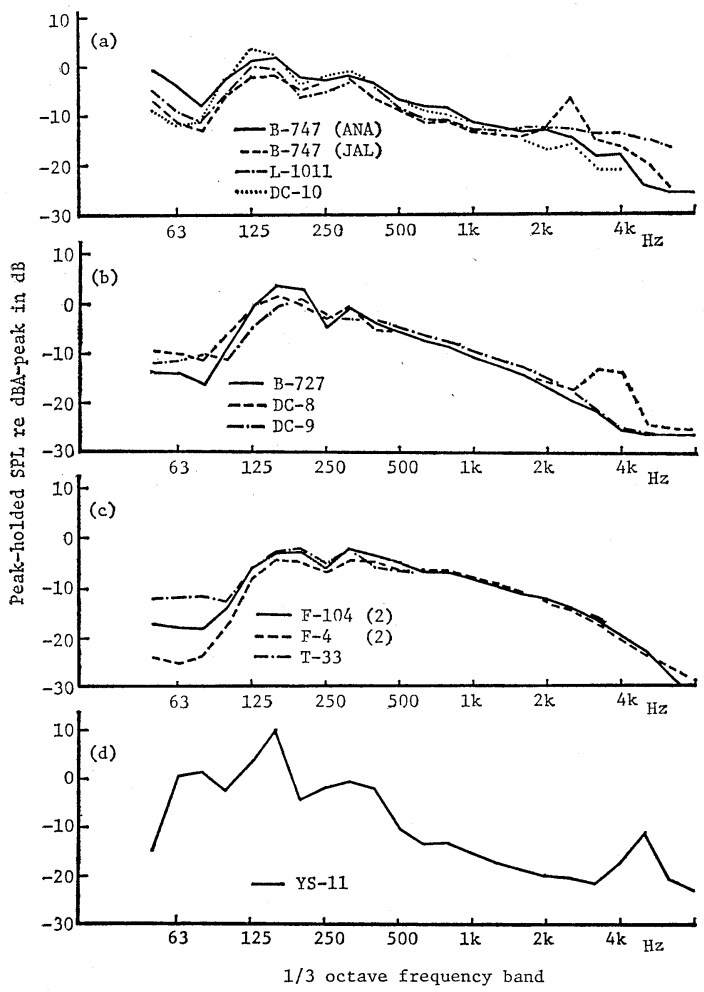

Fig. 7 Superpositions of average peak-hold spectra (one-third octave band) relative to dBA-peak levels for various aircraft types being operated at Chitose airport, Hokkaido; (a) heavy jet airliners having 3 4 high bypass ratio turbo-fan engines, (b) medium jet airliners having $2 \sim 4$ low bypass ratio turbo-fan engines, (c) small jet fighters, and (d) propeller aircraft having 2 turbo-prop engines.
Next, in order to examine the general applicability of the procedure, other field measurements were carried out near Chitose airport, where not only military aircraft but also civil airliners were operated in common. Measurements were made for only Take-off patterns during two days. Microphones were located on the ground, $500 \mathrm{~m}$ from the runway end beneath the flight path. Superpositions of average peak-hold one-third octave band spectra relative to dBA-peaks are shown in Figs. 7 (a) (d). In Fig. 7 (a), B-747 spectra are plotted in two separate lines. Because jet aircraft B-747 are installed with two types of engines and are operated in different conditions by different airlines.

In the middle frequency range $315 \mathrm{~Hz} \sim 2 \mathrm{kHz}$, clear differences can not be inspected among most aircraft types. Especially between military aircraft shown in Fig. 7 (c), differences of spectral characteristics are concentrated in lower frequency bands. So, it was decided to select parameters from lower and higher frequency regions and computed their average values and standard deviations from several data in the first day measurements for each typegroup. They are slightly different from the former case, but the recognition procedure utilized was almost the same. The result shows that the procedure works as effectively as in the former case (see Table 5). There are some wrong classifications among heavy jet airliners with turbo-fan engines of high bypass ratio (B-747, L-1011, DC-10 and A-300) and those among small jet fighters (F-104 and F-4). In fact, these aircraft look alike in their spectral shapes 
Table 5 The result of recognition for Chitose data.

\begin{tabular}{|c|c|c|c|c|c|c|c|c|c|c|c|c|c|}
\hline \multirow[b]{2}{*}{ In } & \multicolumn{13}{|c|}{ Out } \\
\hline & $\begin{array}{l}\text { B-747 } \\
\text { (JAL) }\end{array}$ & $\begin{array}{l}\text { B-747 } \\
\text { (ANA) }\end{array}$ & L-1011 & DC-10 & DC-8 & B-727 & DC-9 & YS-11 & F-104 & $\mathrm{F}-4$ & $\mathrm{~T}-33$ & $\begin{array}{c}\text { Others } \\
\text { (Jet) }\end{array}$ & $\begin{array}{l}\text { Others } \\
\text { (Prop.) }\end{array}$ \\
\hline B-747 JAL & 19 & & 2 & & & & & & & & & & \\
\hline B-747 ANA & & 17 & 2 & & & & & & & & & & \\
\hline L-1011 & & & 8 & & & & & & & & & & \\
\hline DC-10 & & & & 3 & & & & & & & & & \\
\hline DC-8 & & & & & 7 & & & & & & & & \\
\hline B-727 & & & & & & 16 & & & & & & & \\
\hline DC-9 & & & & & & & 16 & & & & 1 & & \\
\hline YS-11 & & & & & & & & 14 & & & & & 1 \\
\hline F-104 & & & & & & & & & 12 & 8 & & & \\
\hline F-4 & & & & & & & & & & 23 & & & \\
\hline $\mathrm{T}-33$ & & & & & & & 1 & & & & 14 & & \\
\hline A-300 & & & 2 & 1 & & & & & & & & & \\
\hline MU-2 & & & & & & & & & & & & & 2 \\
\hline C-1 & & & & & & & 2 & & & & 2 & & \\
\hline F-1 & & & & & & & & & & 2 & & & \\
\hline Others Prop. & & & & & & & & & & & & & 1 \\
\hline
\end{tabular}

(Figs. 7 (a) and (c)).

Finally, one more exercise of the procedure was performed around Osaka international airport, where only civil airliners are operated, in order to confirm that raising the microphone from the ground had little influence upon parameters. Noise measurements were made at four observation points around the airport during a day. Three of the sites, $P t_{1} \sim P t_{3}$ were for $\mathrm{T} / \mathrm{O}$ and the other $P t_{4}$ was for L/D (see Fig. 8). Points $P t_{1}$ and $P t_{4}$ were beneath the flight path close to the runway, $P t_{3}$ was beneath the flight path far from the runway. Point $P t_{2}$ was in the middle of $P t_{1}$ and $P t_{3}$, but was located interior of circular flight path. Microphones were set up at a height of approximately $2 \mathrm{~m}$ above roof tops of buildings. The floor of the roof was surfaced with concrete or asphalt roofing. Used parameters were nearly the same but their values were newly computed from field measurements for each observation point. Agreeable results were obtained through the same recognition procedure (see Table 6) and raising the microphone proved to have little effect on results. Most significant error in the classification was also among heavy jet airliners with turbo-fan engines of high bypass ratio (B-747, L-1011, DC-10 and A-300). Among observation points, results were better at $P t_{1}$ and $P t_{4}$, which were closer to the runway and the flight altitude was not so high.

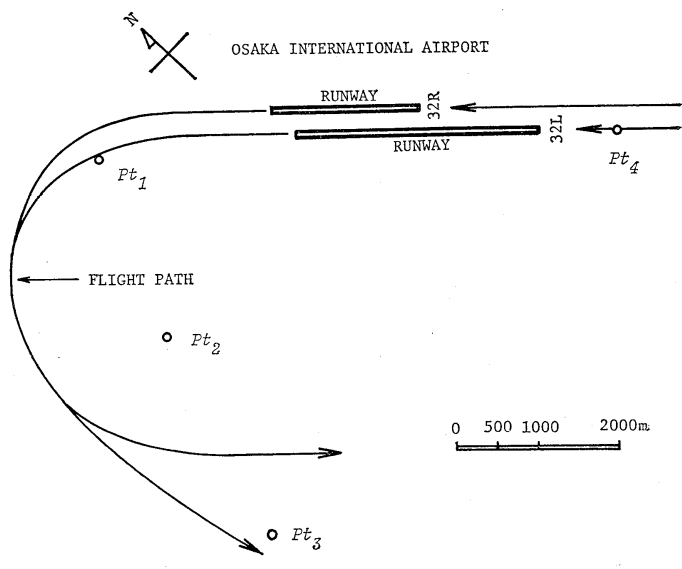

Fig. 8 Geographical locations of observation points in field measurements around Osaka international airport.

\section{CONCLUDING REMARKS}

A method for the acoustic recognition of aircraft types and flight patterns was derived, in which a discriminatory analysis slightly different from the ordinary one was used and peak-hold spectra (onethird octave band) relative to dBA-peak level were selected as characteristic parameters of reference patterns. With a small number of parameters, the 


\section{YAMADA et al.: ACOUSTIC RECOGNITION OF AIRCRAFT TYPES IN FLIGHT}

Table 6 The result of recognition for Osaka data.

\begin{tabular}{lccccc}
\hline \multirow{2}{*}{ Type } & \multicolumn{5}{c}{ Observation point number } \\
\cline { 2 - 5 } & $P t_{1}$ & $P t_{2}$ & $P t_{3}$ & $P t_{4}$ & Total \\
\hline B-747 & $8 / 12$ & $6 / 11$ & $7 / 8$ & $9 / 10$ & $30 / 41$ \\
L-1011 & $13 / 13$ & $13 / 13$ & $8 / 13$ & $11 / 11$ & $45 / 50$ \\
DC-10 & $11 / 12$ & $7 / 10$ & $9 / 10$ & $8 / 8$ & $35 / 40$ \\
A-300 & $5 / 5$ & $0 / 4$ & $1 / 5$ & $2 / 3$ & $8 / 17$ \\
DC-8 & $0 / 1$ & $0 / 1$ & $0 / 1$ & $2 / 2$ & $2 / 5$ \\
B-727 & $10 / 10$ & $10 / 10$ & $9 / 9$ & $10 / 10$ & $39 / 39$ \\
DC-9 & $2 / 2$ & $2 / 2$ & $2 / 2$ & $0 / 1$ & $6 / 7$ \\
YS-11 & $30 / 30$ & $32 / 32$ & $39 / 39$ & $36 / 36$ & $137 / 137$ \\
\hline Total & $79 / 85$ & $70 / 83$ & $75 / 87$ & $78 / 81$ & $302 / 336$ \\
\hline
\end{tabular}

In each column, the left-hand number shows the correct classifications, while the right-hand number is the total observations.

newly developed procedure proved to be able to recognize aircraft types and flight patterns accurately from only acoustic measurements of the aircraft noise. It turned out that the procedure was useful irrespective of the mission of an airfield. Raising the microphone had little effect on the result, but values of characteristic parameters had to be determined in each measuring site, because they were considered to change depending on the geographical relationship between the site and the airport. Moreover, it is quite easy to realize the procedure in a simple and cheap hardware. We have only to prepare a few number of band-pass filters with peakholding circuits and a micro-processor with a little amount of memories, in addition to the common equipment for measuring sound pressure levels.

\section{ACKNOWLEDGEMENTS}

Mr. T. Okamoto of Defence Facilities Environment Improvement Association provided an opportunity to execute the study. Mr. I. Ono et al. of Rion Co. Ltd. cooperated in field measurements and data processing of the final exercise. The authors wish to thank Mr. J. Yoshimura for his assistance in field measurements in the first exercise, and are also grateful to Professor J. Igarashi for his helpful advices and for careful reading of the manuscript.

\section{REFERENCES}

1) G. Nishinomiya, F. Suzuki, and F. Sasaki, "Aircraft noise identification system by correlation technique," IEEE Trans. Broadcast., BC-24 (1978).

2) T. Ono, M. Okuda, I. Ono, N. Hayashi, G. Nishinomiya, F. Suzuki, and F. Sasaki, "Aircraft noise monitoring system with identification system by correlation technique," INTER-NOISE 79, 721724 (1979).

3) Y. Hagino, N. Muranaka, Y. Nomura, and H. Mizutani, "A study on the recognition of aircraft by noise," Reports of the Autumn Meeting, Acoust. Soc. Jpn. (1977), pp. 137-138 (in Japanese).

4) Y. Hagino, Y. Nomura, and H. Mizutani, "A study on the recognition of aircraft by noise," Reports of the Autumn Meeting, Acoust. Soc. Jpn. (1978), pp. 497-498 (in Japanese).

5) A. Nakata, N. Kataoka, and H. Mizutani, "A study on the recognition of aircraft by noise-A majority determination by two dimensional space," Reports of the Spring Meeting, Acoust. Soc. Jpn. (1978), pp. 13-14 (in Japanese).

6) M. G. Kendall, A Course in Multivariate Analysis (Charles Griffin \& Co. Ltd., London, 1968).

7) B. S. Atal, "Automatic recognition of speakers from their voices," Proc. IEEE, 64, 460-475 (1976).

8) I. Yamada, "Cross spectral method for the estimation of noise," J. Acoust. Soc. Jpn. (E) 1, 249-259 (1980).

9) R. C. Payne, "The effect on the measurement of aircraft noise of reflections from ground," NPL Acoustics Report Ac 102 (1982).

10) B. S. Atal, "Automatic speaker recognition based on pitch contours," J. Acoust. Soc. Am. 52, 16871697 (1972). 